\title{
Awareness of Gestational Diabetes among Antenatal Women at The King Fahd Military Medical Complex Hospital in Dhahran, Saudi Arabia Latteefah Saleh Alnaeem \\ College of Applied Medical Sciences, Obstetrics and Gynecology department, King Faisal University, AI Hfouf, Kingdom of Saudi Arabia. \\ *Corresponding author: Latteefah Saleh Alnaeem, E-mail: Latifa_alnaeem@ @otmail.com, Mobile No.: +966504935792, orcid.org/0000-0002-3677-2454
}

\begin{abstract}
Background: Gestational diabetes mellitus (GDM) has recently gained increasing attention among researchers and health professionals. This growing interest is attributed to the rising rate in the development of type 2 diabetes mellitus (DM) during pregnancy and puerperium and later on, as established in GDM patients.

Materials and methods: patients engaged in this survey were informed verbally that their involvement was voluntary, and that completing the distributed questionnaires implied that they had agreed to take part in the study, using questionnaires administered data were gathered by an obstetrics and gynecology doctor, questionnaire was designed following a comprehensive discussion with a team of experts in the field of GDM as it was tested via a pilot study to insure it's reliability. The final copy of the questionnaire that was used in the actual study included 25 questions, a score of one was assigned to each correct answer and zero to each incorrect or "I don't know" answer. A higher score showed that the respondent had better knowledge about GDM.

Conclusion: This research found a considerable lack of awareness about GDM of the pregnant women interviewed. Moreover, the results showed that the respondents were aware that GDM is related to certain factors such as advanced maternal age, overweight, and obesity, among others. It was noted that the lack of awareness and knowledge among pregnant women led to poor self-care and management of GDM, which reflects unmet needs.
\end{abstract}

Keywords: GDM, DM awareness, antenatal, gestational diabetes, Saudi Arabia.

\section{INTRODUCTION}

DM is widely known as being among the most deadly diseases threatening public health globally. Based on statistics from the Center for Disease Control and Prevention, "every five minutes, two people die of diabetes-related causes and 14 adults are newly diagnosed" (1). In Saudi Arabia, the International Diabetes Federation recorded 3.4 million cases of DM in $2015^{(2)}$. The estimated cost of the disease in the country reached $\$ 900$ million in 2010, and is projected increase to more than $\$ 6.5$ billion by 2020 . The rising cost places a tremendous economic burden on the country's economy ${ }^{(3)}$.

Gestational DM (GDM) has recently gained increasing attention among healthcare professionals as a result of growing prevalence of the resultant development of type 2 DM during pregnancy and after birth among GDM patients ${ }^{(3)}$. GDM refers to a lack of carbohydrate tolerance of varying severity with an initial onset during pregnancy, whether or not insulin is used ${ }^{(2)}$. GDM is attributed to various conditions, including an increased rate of preeclampsia and adverse obstetric outcomes that include preterm labor, a high rate of Cesarean sections, and complicated perinatal tears. Other conditions include the possibility of intrauterine fetal death beyond 40 weeks, neonatal hypoglycemia, hypocalcemia, polycythemia, and respiratory distress disease ${ }^{(4)}$. In addition, GDM may affect the offspring's risks of obesity, impaired glucose tolerance, and metabolic syndrome.

A literature review of GDM that was conducted in Saudi Arabia to determine the occurrence of GDM among pregnant women living in the Jizan region also indicated a high prevalence of GDM ${ }^{(5)}$. The study involved 440 pregnant women chosen via a simple random sampling method. The outcome showed that the prevalence of GDM among expectant women was $8.2 \%{ }^{(5)}$. The study also revealed that there was a considerably higher prevalence rate of GDM among overweight women when compared to their counterparts of normal weight. Based on these findings, the authors concluded that the prevalence rate of GDM noted during the study period exceeded the global rate. They also concluded that some of the factors that exposed pregnant women to GDM included neonates weighing more than $3.5 \mathrm{~kg}$, as well as the mother's body mass index (BMI) ${ }^{(5)}$.

In another study, Alfadhli et al. ${ }^{\left({ }^{6}\right)}$ assessed the prevalence of GDM and found that the rate of the disease was $51 \%$ among Saudi mothers in the western region. The study was based on the International Association of the Diabetes and Pregnancy Study Groups' (IADPSG) procedure for screening and diagnosing GDM; hence, the results were valid and relevant to the study.

However, there is little or no knowledge about GDM among expectant Saudi women, 
particularly those who have not yet Been diagnosed with GDM previously ${ }^{(2)}$. Therefore, it is not clear whether or not Saudi mothers who have or who have not had GDM are adhering closely to the optimal GDM guidelines. In addition, Saudi mothers with GDM still need dietary counseling and strategies designed by nutritionists ${ }^{(2)}$.

A contrasting study undertaken by Elmekresh et al. ${ }^{(4)}$ in the Sharjah community in the United Arab Emirates showed that there was a considerable level of awareness about GDM among mothers ${ }^{(4)}$. The purpose of the research was to establish the level of knowledge and awareness of GDM among mothers who were still of childbearing age ${ }^{(4)}$. The target population for this study was women aged 18 to 45 years. The survey involved 450 women. The findings showed that $73.5 \%$ of the respondents were informed about the disorder ${ }^{(4)}$. The study also showed that married women were better informed about the disease than were unmarried ones. The conclusion drawn from the study was that, although women in Sharjah had a high level of awareness, their depth of knowledge about GDM was still poor ${ }^{(4)}$.

Recommendations made following this study were that women in Indian urban areas should be informed about DM in order to be able be monitor their prenatal and antenatal health ${ }^{(7)}$. Despite the fact such an approach is promising in the prevention of GDM, it fails to consider the education level among women in rural areas who are equally exposed to the disease.

A similar study in Malaysia regarding the relationship between better glycemic control among expectant women with GDM and the level of knowledge about GDM showed that women who were aware of GDM could control cases of Their glycaemic levels ${ }^{(8,9)}$. The outcomes of these studies are useful in the assessment of GDM and in the design of follow-up plans during prenatal and postnatal care.

One of the long-standing issues in the management of GDM is the differentiation between overt DM, which goes unnoticed before conception, and the exaggerated gestational variations in the level of glucose metabolism that lead to GDM. According to Facco et al. ${ }^{(10)}$, GDM results from glucose intolerance and occurs mainly during pregnancy. On the other hand, overt DM is the advanced stage DM characterized by the fasting blood glucose concentration. Facco et al. (10) emphasized the need to differentiate between the two to ensure proper management by determining the complications associated with each type. For example, the IADPSG recommends that women should seek a diagnosis and the classification of hyperglycemia following conception. The diagnosis should include an examination of the risk of DM during the first visit for prenatal care. The recommendation is in line with the American Diabetes Association's view, in which the recommended criteria for checkups include this examination earlier in pregnancy ${ }^{(11)}$.

Women should understand and be able to identify risk factors related to GDM. Some of the risk factors to which GDM is linked include a family history of GDM, blood pressure, and a history of diabetes in the family ${ }^{(12)}$. Other factors, as highlighted by Jafari-Shobeiri et al. ${ }^{(12)}$, include cases of congenital abnormality and polycystic ovary disorder, as well as thyroid dysfunction.

Psychological stress has also been linked to the onset of GDM in some women during pregnancy. Martis et al. ${ }^{(13)}$ also linked stress during pregnancy to GMD, arguing that stress can cause expectant women to engage in unhealthy eating, such as consuming foods with high sugar contents, thus raising the level of glucose in the blood and leading to GMD-related complications during pregnancy and after birth.

Notably, knowledge about simple daily practices that can assist in the control or reduction of the number of cases of GDM can make a significant difference in the overall prevalence of GDM among women in Saudi Arabia. Simmons et al. (14) recommend moderate physical activity for expectant mothers to reduce the risk of developing GDM. Simmons et al. ${ }^{(14)}$ explained that physical activity can help to reduce the level of glucose in the blood, which is related to cases of GDM. Simmons et al. ${ }^{(14)}$ recommend walking for at least 25 minutes, four days per week, to control the concentration of excess glucose in the blood.

There are various methods for examining the level of glucose in women during pregnancy. However, the methods vary depending on local guidelines. For example, at the KFMMC, the American College of Obstetrics and Gynecology (15) recommends the administration of a $75-\mathrm{mg}$ oral glucose tolerance test (OGTT) for the diagnosis of GDM.

However, it is important to understand the attitudes of women concerning the management of glucose levels prior to the implementation of practices or drugs to decrease glucose levels. A study undertaken to assess the knowledge and attitudes of expectant mothers toward the oral glucose challenge test in Turkey in 2016 revealed that, in addition to the glucose test, pregnant women requested other tests for various other reasons, including nausea and vomiting, normal test results during a previous pregnancy, or false beliefs regarding the adverse 
effects of the test on them or their unborn babies ${ }^{(7)}$. Such beliefs might be the sources of improper compliance with the glucose test, thus exposing pregnant women to DGM. Therefore, women's perceptions of the OGTT need to be determined in order to ensure that pregnant women comply with the test.

In addition, the patient's understanding of the scale used to manage GDM is essential to ensure that she follows the physicians' treatment instructions strictly once the patient has been diagnosed with GDM. The NICE guidelines for the management of GDM include starting the management with a diet combined with moderate exercise for two weeks. These interventions should be followed by the addition of metformin and, finally, of insulin for the control of the levels of blood sugar ${ }^{(16)}$.

Another major health issue associated with GDM is the development of type 2 DM later in life. In a study to examine the relationship between GDM and type 2 diabetes, Noctor and Dunne ${ }^{(17)}$ found that there was a $60 \%$ chance of someone developing type 2 diabetes following GDM. Other conditions that a woman might develop as a result of GDM include cardiovascular disorder and subclinical atherosclerosis ${ }^{(17)}$. Given these risks and the other complications associated with DM, it is important to assess and enhance the level of awareness among GDM patients regarding the need for follow-up tests to determine the level of glucose during and after the pregnancy.

Breastfeeding has also been found to prevent the development of type $2 \mathrm{DM}$, which in turn causes GDM ${ }^{(18)}$. A study ${ }^{(18)}$ assessing the benefits of breastfeeding among mothers diagnosed with GDM found that breastfeeding can accelerate the metabolism of glucose and lipids for up to three months after pregnancy. Therefore, breastfeeding is a preventive measure for type $2 \mathrm{DM}$, which is a predisposing factor for GDM. In this regard, breastfeeding should be encouraged among mothers to decrease the risks of GDM.

Finally, it is essential to explore the sources of information related to GDM on which expectant women base their actions and attitudes concerning disease screening and management. Such knowledge will help to increase their knowledge about the disease. A study conducted in India to identify leading sources of information regarding GDM among women ${ }^{(7)}$ found that television and radio were the major sources of information about GDM (40\%), followed by doctors (13.3\%), and hospital boards and charts $(18.3 \%){ }^{(1)}$.
Recognition of the sources of information and knowledge related to GDM among women will allow healthcare providers to design a clear database to reduce errors by ensuring that the information offered to the public is in line with the desired or local healthcare guidelines. The strategy can help to prevent GDM, thus enhancing the overall health of the society ${ }^{(19)}$.

In conclusion, the awareness of any disease is the initial step toward preventing it. Therefore, the aim of the current research was to determine the level of awareness among antenatal women in Saudi Arabia via a case study of patients at the KFMMC.

\section{Study Design}

The study used a cross-sectional study design. The survey was undertaken at the antenatal primary care facility of the KFMMC in Dhahran, Saudi Arabia, for eight months from January 2017 to August 2017. A total of 405 eligible patients were engaged in the study. The inclusion criteria included pregnant Saudi women, aged of 16 years and above, who could read. The exclusion criteria were healthcare providers and those with known cases of DM.

\section{Methods}

Permission to undertake this research was sought and approval was granted by the local hospital's Clinical Research Committee. The patients engaged in the survey were also informed verbally that their involvement was voluntary, and that completing the distributed questionnaires implied that they had agreed to take part in the study; the study was conducted on 16/01/2017 at the KFMMC hospital.

The data used in this study were gathered using questionnaires administered by an obstetrics and gynecology doctor, who was the principal investigator. The questionnaire was entirely new, since no questionnaire that related specifically to the evaluation of GDM patients was found. Accordingly, a new questionnaire was designed by gathering some of the questions from previously validated questionnaires covering elements related to the awareness of GDM among mothers receiving antenatal care.

The questionnaire was designed following a comprehensive discussion with a team of experts in the field of GDM, and was then validated by a team of experts including consultants and an associate consultant from the Obstetrics and Gynecology Department at the KFMMC Hospital. Other experts consulted prior to the development of the questionnaire included individuals at the King Faisal University's Obstetrics and Gynecology Department. The content of the questionnaire was 
translated into Arabic to preserve the meaning of important elements captured in the questionnaire. The translated copy of the questionnaire was authenticated in terms of its face and content validity. After the validation and translation of the questionnaire, it was tested via a pilot study that involved 37 patients who were not part of the actual study to ensure its reliability.

The analysis of the reliability of the questionnaire showed a Cronbach's alpha of 0.710 . The final copy of the questionnaire that was used in the actual study included 25 questions grouped into five primary categories that included the patients' basic knowledge of DM and GDM (six questions), the risk factors associated with GDM (five questions), the difference between overt DM and GDM (two questions), the management of GDM (five questions), complications (five questions), and prenatal care and knowledge sources (two questions). The questions had multiple choices, with one "I don't know" option to avoid the respondents guessing.

A score of one was assigned to each correct answer and zero to each incorrect or "I don't know" answer. A higher score showed that the respondent had better knowledge about GDM. The maximum score was 25 , while the minimum was zero $(0)$. The total knowledge score was also grouped as $0-8=$ poor knowledge, 9-16 = fair knowledge, and 17-25 $=$ good knowledge. The percentage of knowledge score was then calculated as the total score obtained divided by the maximum score, which was 25 points.

\section{Ethical Approval}

Ethical approval was obtained from the hospital to carry out the research by administering questionnaires to those receiving healthcare at the KFMMC. The choice of respondents and ethical conduct were created for application in crosssectional research conducted in this healthcare center. Data used in the study were obtained using a self-administered questionnaire designed with the help of an obstetrics and gynecology doctor, who was the principal investigator. The validity of the questionnaire was Ensured using a pilot study, which was conducted on 16/01/2017 at the KFMMC hospital.

\section{Results}

A total of 405 women receiving antenatal care took part in this research. The ages of the participants ranged from 16 to 50 years, with an average age of $28.1 \pm 5.9$ years. With regard to the level of education, $2 \%$ of the participants had low literacy levels, $5.4 \%$ had a primary education, $9.4 \%$ had an intermediate education level, $35.3 \%$ had a secondary education, and $47.9 \%$ had advanced education levels. The majority of the respondents were housewives (86.6\%), with only $13.4 \%$ of them being employed. More than half of those surveyed $(67.2 \%)$ had children, and the rest (32.8\%) were primigravida.

Of the women interviewed, $45.2 \%$ had no family histories of diseases such as DM, GDM, hypothyroidism, hypertension (HTN), or cardiac ailments. However, a family history of DM was noted in $39.8 \%$ of the participants, with a family history of GDM (7.2\%) and a family history of HTN being reported by $27.4 \%$ of the participants. The results further showed that majority of the participants represented by the $76.8 \%$ did not have personal histories of GDM, polycystic ovary syndrome (PCOS), HTN, hypothyroidism, or obesity. However, a few of them (represented by $1.2 \%$ ) disclosed a personal history of DM (see Table $1)$.

With regard to where the antenatal women obtained information about GDM, 45.2\% disclosed that they were not aware of GDM, 24\% had received information from friends or relatives, $15.8 \%$ via social media, $9.6 \%$ from instructional brochures, $7.9 \%$

from books, television, and radio and, finally, 5.4\% from doctors and healthcare providers. It is interesting that $9.9 \%$ of the respondents were not interested in gaining knowledge related to GDM (see Table 1).

Concerning the respondents' knowledge about the risk factors associated with GDM, 75\% of them knew that eating unhealthy food, obesity, and depression Put individuals at risk of GDM, and that they needed to engage in physical exercise in order to avoid GDM. However, only $25.4 \%$ were aware that GDM was related to hypertension.

With regard to awareness about the complications related to GDM, 37\% of the interviewees indicated that they were aware that GDM might lead to cases of low Neonate birth weight, while $62.7 \%$ of them knew that GDM could lead to hidden DM, and $24.9 \%$ acknowledged the possibility of increased risk of congenital anomalies, particularly if DM is not diagnosed. Only $21.7 \%$ of the expectant women respondents knew that untreated DM threatened the health of the unborn child, which might force a termination of the pregnancy, while $45.7 \%$ were unsure about this, and $32.6 \%$ of the women were not aware of such threats.

Concerning the possible future effects of the disease on the baby if the mother has GDM, 50\% of the respondents knew that the child might develop DM in the future as a result of GDM. One quarter of the women knew that there were adverse effects on the baby when the mother develops GDM. Finally, $37.5 \%$ understood that breastfeeding is useful in 
accelerating the metabolism of glucose (see Table 2).

The data presented in Figures 1 illustrate that the GDM knowledge score among participants ranged from $0 \%$ to $95.8 \%$, with an average of $51.5 \pm 17.85 \%$. Fair knowledge of GDM was the highest percentage at $64 \%$, while $17.5 \%$ of the respondents had poor knowledge, and $18.5 \%$ had good knowledge of GDM.

Table 3 indicates that participants with poor knowledge were, on average, younger (26.4 \pm 5.6 years old) than were those with fair or good knowledge about the disorder. In addition, there were higher proportions of poor knowledge among women with primary (41\%) and intermediate levels of education $(44.7 \%)$ than there were among those with secondary school (15.4\%) and advanced levels of education, represented by $10.3 \%$. The differences were statistically significant.

There was a higher proportion of good knowledge about the disease among the working women, represented by $33.3 \%$, compared to knowledge among the housewives, represented by $16.3 \%$. The difference in the knowledge levels between the two groups was statistically significant.

The knowledge levels also differed from one trimester to another. The data obtained indicated that there was also a higher proportion of good knowledge among respondents in their third trimesters $(26 \%)$ than there was in the level of knowledge among those in their first trimesters (12\%). The difference was also statistically significant. Equally importantly, there was a greater proportion of fair knowledge among women with a family history of hypothyroidism (represented by 82.6\%) than there was among respondents without such a history $(56.3 \%)$. As is evident, the difference was statistically significant.

The source of information related to the disorder also determined the level of knowledge among the patients. The finding showed that there was a higher proportion of good knowledge (36.4\%) among the respondents who obtained the information from healthcare providers than there was among those who were not concerned about the information. There was also a considerably higher level of fair knowledge (76.3\%) among pregnant women who learned about the disorder from their friends or relatives than there was among the respondents who did not mention having any source of such information (represented by 56.8\%). However, no statistically significant variations were observed among the factors assessed in Table 3.

The knowledge score percentage was entered as a dependent variable into the multiple linear regression while controlling for other factors including the age, level of education, employment status, and trimester. On the other hand, a positive family history of medical disorders was entered as an independent factor. Pregnant women whose relatives had issues with glucose intolerance were at risk of developing GDM; thus, they should be diagnosed on time. The findings revealed that a higher knowledge score was attributed to factors such as older age, higher levels of education, working, multi-parity, and to those with positive family histories of disease, particularly thyroid dysfunction (see Table

4).

Table 1 -Sociodemographic and gynecological histories of the participants and their information sources.

\begin{tabular}{|llll|}
\hline & & \multicolumn{2}{l|}{$\begin{array}{l}\text { No. } \\
(\mathrm{n}=405)\end{array}$} \\
\hline \hline Age (in years) & Min-max & \multicolumn{2}{c|}{$16-50$} \\
& Mean \pm SD & $28.10 \pm 5.90$ \\
\hline \multirow{3}{*}{ Education level } & Illiterate & 8 & 2.00 \\
& Primary school & 22 & 5.40 \\
& Intermediate school & 38 & 9.40 \\
& Secondary school & 143 & 35.30 \\
\multirow{2}{*}{ Occupation } & Higher education & 194 & 47.90 \\
\hline \multirow{2}{*}{ Parity } & Housewife & 350 & 86.60 \\
& Working & 54 & 13.40 \\
\hline \multirow{2}{*}{ Trimester } & No children & 133 & 32.80 \\
& Has children & 272 & 67.20 \\
\hline
\end{tabular}

Table 1 Cont. below 
Table 1 Cont.

\begin{tabular}{|lll|}
\hline & & No. $(\mathrm{n}=405)$ \\
\hline \hline \multirow{5}{*}{ Family history } & None & 183 \\
& DM & 161 \\
& GDM & 32 \\
& HTN & 111 \\
& Hypothyroidism & 46 \\
& Cardiac disease & 41 \\
\hline \multirow{5}{*}{ Personal history } & None & 311 \\
& DM & 5 \\
& GDM & 36 \\
& HTN & 19 \\
& Hypothyroidism & 27 \\
& PCOS & 6 \\
Obesity & 33 \\
\hline \hline \multirow{3}{*}{ Source of } & Do not care to know & 40 \\
information & No idea about GDM & 183 \\
& Previous GDM & 29 \\
& Friends or relatives & 97 \\
& Books/newspaper/magazine & 32 \\
TV/radio & 32 \\
& Healthcare provider & 22 \\
& Instructional brochures & 39 \\
Social media & 64 \\
\hline
\end{tabular}

SD: standard deviation, DM: diabetes mellitus, GDM: gestational DM, HTN: hypertension, PCOS: polycystic ovary syndrome

Table 2 - Distribution of pregnant women based on their awareness of the risk factors related to GDM, complications, and the relationship between breastfeeding and GDM.

\begin{tabular}{|c|c|c|c|c|c|c|}
\hline \multirow{2}{*}{ Information about GDM } & \multicolumn{2}{|c|}{ I don't know } & \multicolumn{2}{|l|}{ No } & \multicolumn{2}{|l|}{ Yes } \\
\hline & No. & $\%$ & No. & $\%$ & No. & $\%$ \\
\hline Risk factors & & & & & & \\
\hline Obesity/overweight & 45 & 11.10 & 45 & 11.10 & 315 & $77.8^{\vee}$ \\
\hline Stress/depression & 78 & 19.30 & 37 & 9.10 & 290 & $71.6^{\vee}$ \\
\hline Exercise & 62 & 15.30 & 24 & 5.90 & 319 & $78.8^{\vee}$ \\
\hline Unhealthy food & 50 & 12.30 & 31 & 7.70 & 324 & $80.0^{\vee}$ \\
\hline Hypertension & 173 & 42.70 & 129 & 31.90 & 103 & $25.4^{\sqrt{ }}$ \\
\hline Complications & & & & & & \\
\hline Increased baby birth weight & 134 & 33.10 & 121 & 29.90 & 150 & $37.0^{\vee}$ \\
\hline Possibility of hidden DM & 118 & 29.10 & 33 & 8.10 & 254 & $62.7^{\vee}$ \\
\hline $\begin{array}{l}\text { Increased risk of congenital anomalies in cases of } \\
\text { undiagnosed DM }\end{array}$ & 185 & 45.70 & 119 & 29.40 & 101 & $24.9^{\vee}$ \\
\hline $\begin{array}{l}\text { No potential for the need to terminate pregnancy in } \\
\text { cases of uncontrolled GDM }\end{array}$ & 185 & 45.7 & 88 & $21.7^{\vee}$ & 132 & 32.6 \\
\hline $\begin{array}{l}\text { Future childhood effects on babies of GDM } \\
\text { mothers }\end{array}$ & 149 & 36.8 & 158 & 39.0 & 98 & $24.2^{\vee}$ \\
\hline Future DM as consequence of GDM & 122 & 30.1 & 80 & 19.8 & 203 & $50.1^{\vee}$ \\
\hline $\begin{array}{l}\text { Breastfeeding has effects on improving glucose } \\
\text { metabolism }\end{array}$ & 205 & 50.6 & 48 & 11.9 & 152 & $37.5^{\vee}$ \\
\hline There was a preconception about GDM counseling & 95 & 23.5 & 206 & 50.9 & 104 & $25.6^{\vee}$ \\
\hline
\end{tabular}

SD: standard deviation, DM: diabetes mellitus, GDM: gestational DM 
Table 3 - Correlation between knowledge about GDM among pregnant women and potentially related factors.

\begin{tabular}{|c|c|c|c|c|c|c|c|c|}
\hline & \multicolumn{6}{|c|}{ Knowledge } & \multirow{3}{*}{$\begin{array}{l}\text { Test of } \\
\text { significance } \\
\mathrm{X}^{2}, \mathrm{p}\end{array}$} \\
\hline & & \multicolumn{2}{|c|}{ Poor } & \multicolumn{2}{|l|}{ Fair } & \multicolumn{2}{|c|}{ Good } & \\
\hline & & No & $\%$ & No. & $\%$ & No & $\%$ & \\
\hline $\begin{array}{l}\text { Age } \\
\text { (in years) }\end{array}$ & $\begin{array}{l}(\text { Min-max }) \\
\text { Median } \\
\text { Mean } \pm \text { SD }\end{array}$ & \multicolumn{2}{|c|}{$\begin{array}{l}16-40 \\
27 \\
26.4 \pm 5.6\end{array}$} & \multicolumn{2}{|c|}{$\begin{array}{l}17-46 \\
27 \\
28.1 \pm 5.6\end{array}$} & \multicolumn{2}{|c|}{$\begin{array}{l}19-50 \\
29 \\
29.6 \pm 6.6\end{array}$} & $\begin{array}{c}\mathrm{F}=5.7 \\
\mathrm{p}=0.004\end{array}$ \\
\hline \multirow{5}{*}{ Education level } & Illiterate & 3 & 37.50 & 3 & 37.50 & 2 & 25.00 & \multirow{5}{*}{$\begin{array}{l}38.2, \\
\mathrm{p}<0.0001\end{array}$} \\
\hline & Primary school & 9 & $40.9 \mathrm{a}$ & 10 & 45.50 & 3 & 13.60 & \\
\hline & Intermediate school & 17 & $44.7 \mathrm{a}$ & 17 & 44.70 & 4 & 10.50 & \\
\hline & Secondary school & 22 & $15.4 b$ & 94 & 65.7 & 27 & 18.9 & \\
\hline & Higher education & 20 & $10.3 b$ & 135 & 69.6 & 39 & 20.1 & \\
\hline \multirow{2}{*}{ Occupation } & Housewife & 68 & $19.4 \mathrm{a}$ & 225 & 64.3 & 57 & $16.3 \mathrm{a}$ & \multirow[t]{2}{*}{$12.5,0.002$} \\
\hline & Working & 3 & $5.6 \mathrm{~b}$ & 33 & 61.1 & 18 & $33.3 b$ & \\
\hline \multirow[t]{2}{*}{ Parity } & No children & 35 & $26.3 \mathrm{a}$ & 83 & 62.4 & 15 & $11.3 \mathrm{a}$ & \multirow[t]{2}{*}{$14.4,0.001$} \\
\hline & Has children & 36 & $13.2 \mathrm{~b}$ & 176 & 64.7 & 60 & $22.1 \mathrm{~b}$ & \\
\hline
\end{tabular}

Table 3 Cont.

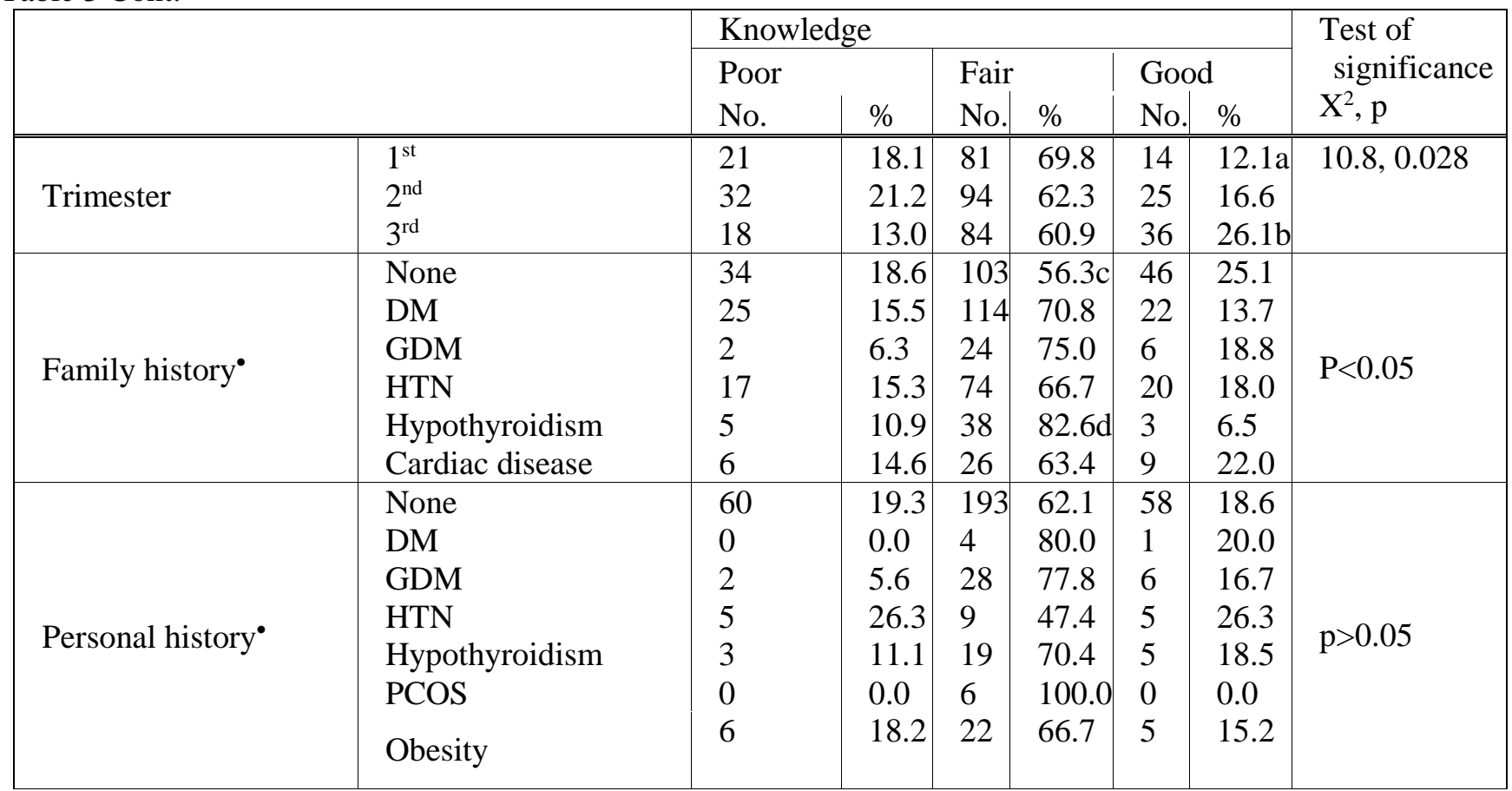

Table 3 Cont.

\begin{tabular}{|c|c|c|c|c|c|c|c|c|}
\hline & & \multicolumn{6}{|c|}{ Knowledge } & \multirow{3}{*}{$\begin{array}{l}\text { Test of } \\
\text { significance } \\
\mathrm{X}^{2}, \mathrm{p}\end{array}$} \\
\hline & & \multicolumn{2}{|c|}{ Poor } & \multicolumn{2}{|l|}{ Fair } & \multicolumn{2}{|c|}{ Good } & \\
\hline & & No. & $\%$ & No. & $\%$ & No. & $\%$ & \\
\hline \multirow{9}{*}{$\begin{array}{l}\text { Source of } \\
\text { information }\end{array}$} & Do not care to know & 8 & 20.0 & 30 & 75.0 & 2 & $5.0 \mathrm{c}$ & \multirow{9}{*}{$\mathrm{P}<0.05$} \\
\hline & No & 45 & 24.6 & 104 & $56.8 \mathrm{c}$ & 34 & 18.6 & \\
\hline & Previous GDM & 2 & 6.9 & 22 & 75.9 & 5 & 17.2 & \\
\hline & Friends or relatives & 9 & 9.3 & 74 & $76.3 \mathrm{~d}$ & 14 & 14.4 & \\
\hline & Books/newspaper/magazine & 3 & 9.4 & 21 & 65.6 & 8 & 25.0 & \\
\hline & TV/radio & 1 & 3.1 & 24 & 75.0 & 7 & 21.9 & \\
\hline & Healthcare provider & 2 & 9.1 & 12 & 54.5 & 8 & $36.4 \mathrm{~d}$ & \\
\hline & Instructional brochures & 7 & 17.9 & 22 & 56.4 & 10 & 25.6 & \\
\hline & Social media & 4 & 6.3 & 44 & 68.8 & 16 & 25.0 & \\
\hline
\end{tabular}

SD: standard deviation, DM: diabetes mellitus, GDM: gestational DM, HTN: hypertension, PCOS: polycystic ovary syndrome,

- Data are not mutually exclusive. Row \% was calculated from the number of cases answered.

${ }^{a}$ and ${ }^{b}$ are significantly different at $\mathrm{p}<0.05$ in the two-sided test of the equality of proportions ( $\mathrm{z}$ test), adjusted for all pairwise comparisons using the Bonferroni correction.

${ }^{\mathrm{c}}$ and ${ }^{\mathrm{d}}$ are significantly different at $\mathrm{p}<0.05$ in the two-sided test of the equality of proportions ( $\mathrm{z}$ test). 
Table 4 - Multiple linear regression for factors linked to a higher GDM knowledge score.

\begin{tabular}{|l|l|l|l|l|l|}
\hline \multirow{2}{*}{ Factor } & \multicolumn{2}{|l|}{$\begin{array}{l}\text { Unstandardized } \\
\text { coefficients }\end{array}$} & $\begin{array}{l}\text { Standardized } \\
\text { coefficients }\end{array}$ & $\mathrm{T}$ & $\mathrm{p}$ \\
\cline { 2 - 5 } & $\mathrm{B}$ & Standard error & Beta & & \\
\hline (Constant) & 3.396 & 6.297 & & .539 & .590 \\
Age & .491 & .162 & .162 & 3.024 & .003 \\
Education level & 4.012 & .907 & .213 & 4.425 & .000 \\
Working mother & 5.761 & 2.518 & .110 & 2.288 & .023 \\
Parity & 7.106 & 2.027 & .187 & 3.505 & .001 \\
Trimester 2 & .233 & 2.058 & .006 & .113 & .910 \\
Trimester 3 & 3.589 & 2.106 & .095 & 1.704 & .089 \\
Positive family history of & 4.263 & 1.693 & -.119 & -2.518 & .012 \\
medical diseases & & & & & \\
\hline
\end{tabular}

$\mathrm{F}=10.39, \mathrm{p}=0.0001, \mathrm{R}^{2}=15.5 \%$

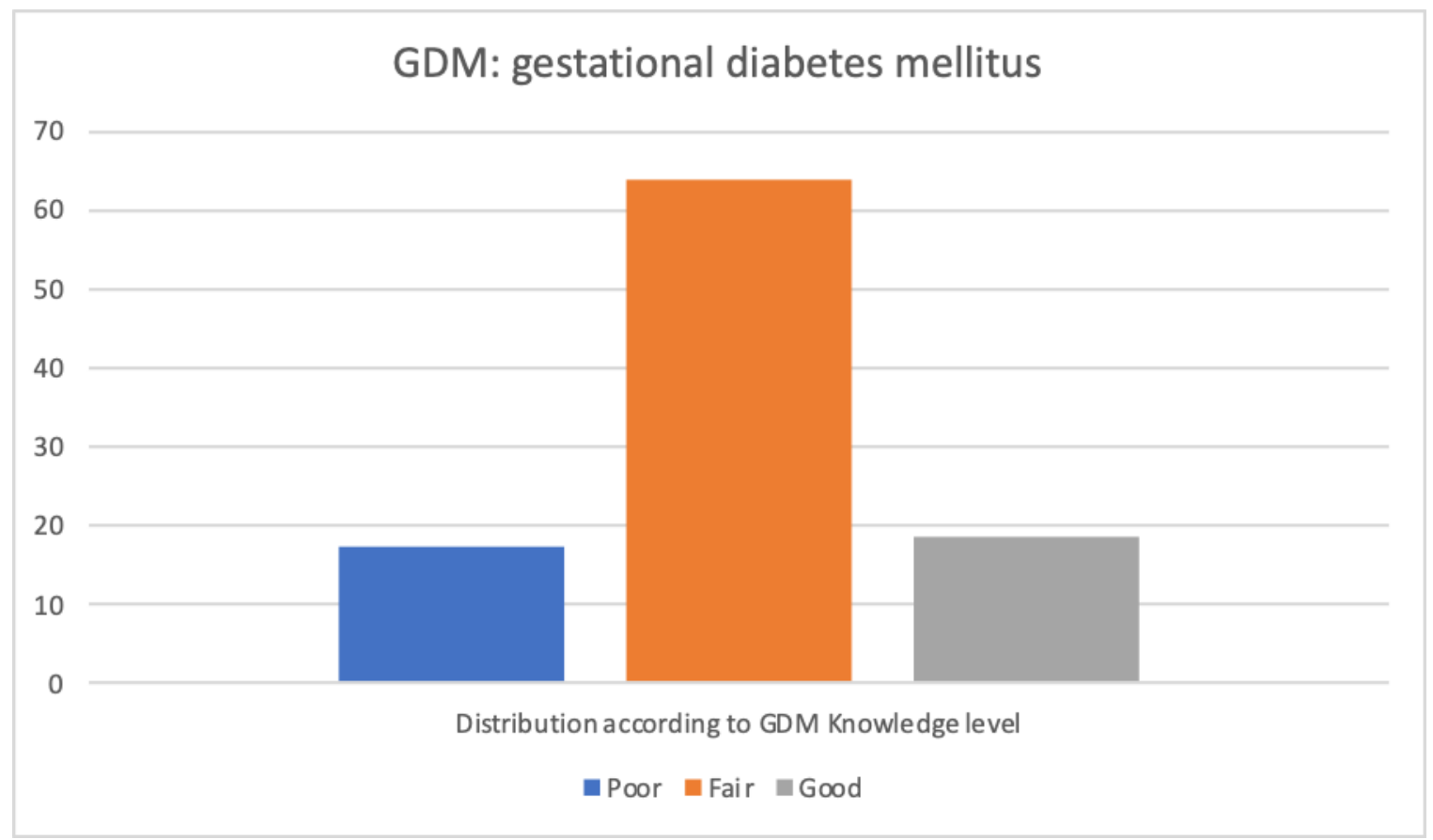

\section{Figure 1- GDM Knowledge level}

\section{DISCUSSION}

The research report examined 405 pregnant women regarding their awareness of GDM. Those engaged in the study were aged between 16 and 50, with an average age of $28.1 \pm 5.9$ years. With regard to education, the majority of the participants $(47.9 \%)$ had a high level of education, $35.3 \%$ had a secondary education level, 9.4\% had an intermediate education level, $5.4 \%$ had a primary education level, and $2 \%$ were illiterate. Therefore, a significant number of the respondents had basic knowledge and could read. However, there was higher proportion of poor knowledge among the participants with primary education $(41 \%)$ and intermediate education levels $(44.70 \%)$ than there was among those with secondary school $(15.40 \%)$ and advanced education levels $(10.30 \%)$. The results indicate substantial statistical differences.

The findings illustrated that nearly half (45.2\%) of the participants did not have family histories of hypothyroidism, HTN, DM, GDM, or cardiac disorders. However, some respondents had family histories of DM (39.80\%), HTN (27.40\%), hypothyroidism (11.40\%), cardiac disease (10.10\%), and GDM (7.2\%). Similarly, the majority of the participants (represented by $76.80 \%$ ) did not have personal histories of disorders such as GDM, hypothyroidism, HTN, PCOS, or obesity. Only $8.90 \%$ of them reported personal histories of GDM. Some studies ${ }^{(21,22,23,24)}$ have revealed that a family history of DM is related to a greater risk of an individual developing GDM.

The study indicated that $45.2 \%$ of those interviewed lacked knowledge about GDM, while $24 \%$ had obtained information about GDM from relatives or friends, $15.80 \%$ via social media, and $5.40 \%$ from healthcare providers. The rest of the respondents obtained information regarding the disorder from television, instructional brochures, 
books, and radio, while $9.90 \%$ were not interested in learning about it.

Improved health literacy is important for one's understanding and choice of a healthy lifestyle. Moreover, the efficient management of GDM requires an individual to seek a timely diagnosis of the condition, which might be challenging because such a decision requires an increase in health literacy skills and knowledge in order for an individual to understand the roles that screening and management play in GDM during the initial treatment window (25). Damm et al. (26) emphasized that a lack of knowledge about GDM might lead to a delayed diagnosis or failure to comply with recommended medication, thus contributing to poor management of the condition. Therefore, the authors recommended educating women to ensure that they sought a diagnosis before conception (26).

Knowledge regarding the risk factors associated with GDM was also assessed because this determines an individual's ability to prevent or manage the condition. The results showed that more than $75 \%$ of the respondents were aware that the consumption of unhealthy food, obesity, and depression (71.6\%) increased the risk of developing GDM, and that women needed to exercise before and after pregnancy in order to reduce the risk of developing the condition. However, only a quarter $(25.40 \%)$ of the respondents knew that GDM was related to hypertension.

Simmons et al. ${ }^{(27)}$ concurred with Damm et al. ${ }^{(25)}$ by recommending maternal education to decrease the risk of developing GDM. Simmons et al. ${ }^{(27)}$ also suggested maternal education to avoid unhealthy behaviors such as the consumption of foods rich in saturated fats, red or processed meats, and high glycogenic loads, which might lead to issues such as obesity, which might also Lead to an increased risk of developing GDM. However, diets rich in polyunsaturated fats, carbohydrates, and fiber have protective effects against the development of GDM.

The research revealed that $37 \%$ of the women involved in this study were aware that GDM might lead to an increase in birth weight, $62.7 \%$ knew about the possibility of hidden DM, and $24.90 \%$ were aware of the possibility of the increased risk of congenital anomalies in the cases of undiagnosed DM. Simmons et al. (27) also attributed GDM to abnormal BMI, a family history of diabetes, and a high birth weight. Only $21.70 \%$ of the pregnant women interviewed were aware that delayed treatment or untreated DM posed a health risk to the unborn child, which might necessitate a termination of the pregnancy. On the other hand, $45.70 \%$ of the interviewees were not sure about the risks and $32.60 \%$ were not aware of them. These results are in line with Hussain et al. ${ }^{(28)}$ view that more than one quarter of pregnant women rarely seek a timely diagnosis of DM, implying that they do not understand the risks.

According to Hussain et al.$^{(28)}$, pregnant women with low education levels tended to be least knowledgeable about GDM; the authors Hussain et al.$^{(28)}$ concluded that low literacy in relation to diabetes caused poor management and self-care among women with GDM. Therefore, the level of education has a significant impact on a patient's health literacy.

With regard to future impacts on the child of a mother with GDM, half of the respondents acknowledged that they were aware of possible future DM as a result of untreated GDM. Further results indicated that $25 \%$ of the interviewees acknowledged that GDM might lead to adverse effects during childhood, while $37.5 \%$ knew that breastfeeding can enhance the metabolism of glucose. Simmons et al. ${ }^{(27)}$ reiterated that pregnant women with a family history of DM risked developing GDM; hence, they should seek a timely diagnosis. Remarkably, the lack of such a relationship was linked to the low statistical power or the few positive cases of GDM.

\section{Acknowledgments:}

I would like to express my deepest appreciation to all those who provided me the possibility to complete this research from family to friends.

\section{REFERENCES}

1. the centers for disease control (C.D.C) and prevention, (2018): Diabetes-data and statistics. Available

https://www.cdc.gov/diabetes/data/index.html

2. Hussain $Z$ (2015): Evaluation of knowledge regarding gestational diabetes mellitus and its association with glycaemic level: A Malaysian study. Primary Care Diabetes, 9(3):184-190.

3. Facco F L (2017): Objectively measured short sleep duration and later sleep midpoint in pregnancy are associated with a higher risk of gestational diabetes. American Journal of Obstetrics and Gynecology, 217(4): 447-465.

4. Elmekresh A (2017): Gestational diabetes awareness in women of childbearing age in Sharjah. Glob J Obes Diabetes Metab Syndr., 4(2):51-53.

5. Abdelmola A O (2017): Gestational diabetes prevalence and risk factors among pregnant womenJazan Region, Saudi Arabia. Clinical Diabetology, 6(5):172-177.

6. Alfadhli E M (2015): Gestational diabetes among Saudi women: prevalence, risk factors and pregnancy outcomes. Annals of Saudi Medicine, 35(3):222. 
7. Shriraam V (2013): Awareness of gestational diabetes mellitus among antenatal women in a primary health center in South India. Indian Journal of Endocrinology and Metabolism, 17(1):146-148.

8. Syed Y (2016): A study to determine awareness of gestational diabetes mellitus amongst pregnant women coming for ante natal care in a medical college hospital situated in rural area of Jaipur. Indian J Appl Res., 6(1):12-33.

9. Wander P (2016): To Study the Awareness about Gestational Diabetes Mellitus in Females among General Population. Journal of Clinical Diabetology, $3(1): 1-28$.

10. Facco F L (2017): Objectively measured short sleep duration and later sleep midpoint in pregnancy are associated with a higher risk of gestational diabetes. American Journal of Obstetrics and Gynecology, 217(4):447-465.

11.ADA criteria (2017): American Diabetes Association. 2. Classification and diagnosis of diabetes. Diabetes Care, 40:S11.

12.Jafari-Shobeiri M (2015): Prevalence and risk factors of gestational diabetes in Iran: a systematic review and meta-analysis. Iranian Journal of Public Health, 44(8):1036-1050.

13. Martis R (2018): Enablers and barriers for women with gestational diabetes mellitus to achieve optimal glycaemic control-a qualitative study using the theoretical domains framework. BMC Pregnancy and Childbirth, 18(1):91.

14. Simmons D (2016): Effect of physical activity and/or healthy eating on GDM risk: the DALI lifestyle study. The Journal of Clinical Endocrinology \& Metabolism, 102(3):903-913.

15.American College of Obstetricians and Gynecologists (2013): Practice bulletin no. 137: Gestational diabetes mellitus. Obstetric Gynecol., 122(1):406-16.

16. National Collaborating Centre for Women's and Children's Health UK. Diabetes in pregnancy (2015):

https://www.ncbi.nlm.nih.gov/books/NBK328422/

17. Noctor $\mathbf{E}$ and Dunne F P (2015): Type 2 diabetes after gestational diabetes: the influence of changing diagnostic criteria. World Journal of Diabetes, 6(2): pp. 234-240.
18. Much D (2014): Beneficial effects of breastfeeding in women with gestational diabetes mellitus. Mol Metab., 3(3):284-292.

19.American Diabetes Association (2015): Classification and diagnosis of diabetes. Diabetes Care, 38(1):8-16.

20.Frank Wolf M (2017): Isolated polyhydramnios in the third trimester: is a gestational diabetes evaluation of value? Gynecological Endocrinology, 33(11): 849852.

21.Seshiah V (2008): Prevalence of gestational diabetes mellitus in South India (Tamil Nadu) - a community based study. J Assoc Physicians India, 56:329-33.

22.Zargar A H (2004): Prevalence of gestational diabetes mellitus in Kashmiri women form the Indian subcontinent. Diabetes Res Clin Pract., 66 (2): 139 145

23.Swami S R (2008): Prevalence of Carbohydrate intolerance of varying degrees in pregnant females in western India (Maharastra) - a hospital based study. J Idnian Med Assoc.,106(11): 712-4, 735

24. Kim C (2009): Does frank diabetes in first degree relatives of a pregnant woman affect the likelihood of her developing gestational diabetes mellitus or nongestational diabetes? Am J Obstet Gynecol., 201(576):e1-6.

25.Hartling L (2013): Benefits and harms of treating gestational diabetes mellitus: a systematic review and meta-analysis for the US Preventive Services Task Force and the National Institutes of Health Office of Medical Applications of Research. Annals of Internal Medicine, 159(2):123-129.

26.Damm P (2016): Gestational diabetes mellitus and long-term consequences for mother and offspring: a view from Denmark. Diabetologia, 59(7):1396-1399.

27.Simmons D (2015): Results from a European multicenter randomized trial of physical activity and/or healthy eating to reduce the risk of gestational diabetes mellitus: the DALI lifestyle pilot. Diabetes Care, 38(9):1650-1656.

28. Hussain $Z$ (2015): Evaluation of knowledge regarding gestational diabetes mellitus and its association with glycaemic level: A Malaysian study. Primary Care Diabetes, 9(3):7184-190. 